\title{
A Review of Minimally Invasive Strabismus Surgery (MISS): Is This the Way Forward?
}

Ioannis Asproudis · Nikolaos Kozeis · Andreas Katsanos •

Saurabh Jain · Paris G. Tranos · Anastasios-Georgios P. Konstas

Received: January 16, 2017 / Published online: March 1, 2017

(C) The Author(s) 2017. This article is published with open access at Springerlink.com

\begin{abstract}
Minimally invasive surgery is rapidly becoming the norm in medicine, as it often leads to better outcomes and earlier rehabilitation. This article reviews the principles and different techniques employed to perform minimally invasive strabismus surgery (MISS). In these techniques, strabismus surgery is performed through keyhole openings, thus reducing the risk of postoperative corneal complications, minimizing postoperative discomfort, and better preserving muscle function. MISS can be used to perform all types of strabismus surgery, namely rectus muscle recessions, resections, plications,
\end{abstract}

Enhanced content To view enhanced content for this article go to http://www.medengine.com/Redeem/ FF87F06054145228.

I. Asproudis - A. Katsanos

Department of Ophthalmology, University of

Ioannina, Ioannina, Greece

N. Kozeis · P. G. Tranos

Ophthalmica Institute, Thessaloniki, Greece

S. Jain

Royal Free London NHS Trust, University College

London, London, UK

A.-G. P. Konstas ( $\square)$

1st and 3rd University Departments of Ophthalmology, Aristotle University of

Thessaloniki, AHEPA Hospital, 1 Kyriakidi Street,

54636 Thessaloniki, Greece

e-mail: konstas@med.auth.gr reoperations, retroequatorial myopexy, transpositions, oblique muscle recessions, or plications even in the presence of limited motility. Of note, ocular alignment outcomes with MISS versus more traditional techniques have not been compared in randomized trials. Consequently, more controlled evidence is still needed to better delineate the future role and value of MISS in clinical management.

Keywords: Conjunctival incisions; Minimally invasive strabismus surgery; MISS; Ophthalmology; Strabismus surgery; Squint surgery

\section{INTRODUCTION}

Minimal access surgery has gained popularity in many fields of ophthalmology. Current and emerging procedures like phacoemulsification, sutureless vitrectomies, minimally invasive lid surgery, and miniature, novel glaucoma stents and implants are in essence minimally invasive surgical therapies allowing earlier rehabilitation. A similar approach to strabismus surgery offering less postoperative discomfort and equally successful outcomes compared to conventional strabismus surgery may provide a worthwhile alternative. The choice of conjunctival incision is an important step in strabismus surgery. Various techniques have been 
described, offering appropriate access and enhanced exposure of the extraocular muscles. The current paper reviews key available literature on minimally invasive approaches in strabismus surgery. This article is based on previously conducted studies and does not involve any new studies of human or animal subjects performed by any of the authors.

Swan and Talbott [1] described an incision just behind the insertion of the recti muscles. However, this technique is now used primarily for the vertical recti muscles, especially for the superior rectus, to preserve conjunctival tissue for possible future operations (e.g., glaucoma filtering surgery).

Harms described a limbal approach [2, 3]. This technique permitted full visualization of the muscle undergoing operation and avoided excessive scarring and bleeding over the muscle tendon. Some common postoperative complications included discomfort, interpalpebral conjunctival redness, corneal dellen, and Tenon's capsule prolapse.

Parks described an alternative fornix approach [4]. Although this technique decreases postoperative discomfort, it is difficult to perform in children because of their prominent Tenon's capsule, in cases with significant preexisting scarring, and in older patients with inelastic conjunctiva.

Velez described single snip surgery with the incision placed radially [5], while Gobin and Bierlaagh [6] later developed a technique for accessing the recti through two small radial openings, one along the superior and the other along the inferior muscle margin to perform hang-back recessions.

Mojon in 2007 modified this approach further, designating this technique minimally invasive strabismus surgery (MISS) [7]. This method involves performing strabismus surgery through keyhole openings to decrease tissue trauma, minimize postoperative complications and patient discomfort, and improve surgical outcomes.

The basic principles underlying MISS are

- Use of an operating microscope Although MISS can also be performed using magnifying loupes, it has been suggested that the higher magnification offered by the operating microscope will allow smoother tissue handling during dissection, easier detection of anatomical abnormalities, and better control of bleeding [8].

- Use of traction sutures The muscle undergoing operation is exposed with the use of a corneal traction suture (e.g., 6-0 Prolene). In most cases the exposure is adequate so that an assistant may not be needed. Should the exposure using the corneal traction suture prove inadequate, two additional scleral traction sutures can be tried. These should be placed on either side of the muscle insertion after the two parainsertional conjunctival cuts are fashioned.

- Creation of appropriately placed keyhole openings The openings are placed as far away from the limbus as possible. Conjunctival openings that are not necessary for the performance of the surgical steps should be avoided. The keyhole conjunctival cuts are placed in a fashion that allows their extension. For example, the two parainsertional cuts used in rectus surgery can be extended anteriorly and joined at the limbus to create a conventional Harm's opening. Although the small keyhole incisions can be left unsutured at the completion of the case, it is probably better to close them with one or two 8-0 or 9-0 absorbable wing sutures so that postoperative discomfort and the risk of infection are further minimized. As the incisions are located at the periphery and hence normally covered by the eyelids, foreign body sensation and redness in the postoperative period are minimized.

- Creation of sub-Tenon's tunnels Tunnels between the two parainsertional conjunctival incisions allow the surgeon to isolate the muscle insertion before recessing or advancing the muscle. Additionally, tunnels may allow access to more posterior parts of the muscle or the perimuscular tissue.

- Minimization of perimuscular tissue disruption Dissection of perimuscular tissue is reduced to the absolute minimum that will still allow the surgeon to displace or anchor a muscle. Limiting all unnecessary tissue handling ensures that iatrogenic trauma is minimized 
and hence postoperative complaints are generally mild. Importantly, by diminishing tissue trauma we also reduce the chances of significant subconjunctival fibrosis.

- Transconjunctival suturing techniques Techniques have been described for suturing muscles or for reattaching muscles to sclera through minimally dissected conjunctiva $[8,9]$. These techniques allow the surgeon to perform many procedures during MISS without the need to extend the incisions, or the area of dissection, thus minimizing collateral tissue damage.

A number of potential short- and long-term benefits of MISS render the technique an attractive surgical option for many patients:

1. Reduction of conjunctival congestion and chemosis $[8,10]$ One of the benefits of minimizing surgical trauma after MISS is that patients normally have less severe conjunctival trauma, and thus less discomfort and more acceptable cosmetic appearance in the early postoperative period (Figs. 1, 2). Because the MISS incisions are very small and placed away from the limbus so that the eyelids normally cover them, the resultant foreign body sensation caused by the cuts and/or the stitches is in most cases negligible. Another advantage of the incisions being placed away from the cornea is that complications such as dellen or dry eye are avoided. Moreover, the reduced chemosis and the fact that the limbus is uninvolved may allow earlier introduction of contact lenses.

2. Decreased scarring of the perimuscular tissue This long-term benefit of the diminished surgical trauma may prove particularly important should reoperation become necessary.

3. Preservation of perilimbal episcleral vessels As perilimbal blood vessels remain intact following MISS, the risk of postoperative anterior segment ischemia is greatly reduced compared to the conventional surgical techniques that require dissection of the limbal conjunctiva [11].

On the other hand, the disadvantages of MISS should be acknowledged:

1. The technique is initially technically challenging. Therefore, surgeons wishing to incorporate MISS in their armamentarium may need to start with easier more straightforward cases and gradually proceed to more demanding ones. Furthermore, assisting an experienced MISS surgeon in a few cases will allow a smoother introduction to MISS.

2. Surgical time is longer, at least for the surgeon who is unfamiliar with MISS $[8,10,12,13]$. With increasing experience, MISS should last less than conventional surgery. This is especially true for rectus muscle transpositions because large openings are not needed [8].

3. Although published evidence is inconclusive, it has anecdotally been suggested that the risk of scleral perforations is increased with MISS [8].

Challenges and complications common in MISS have been described [8]. Tearing of a keyhole cut that involves Tenon's capsule may result in visible scar. If the tear occurs over a muscle then
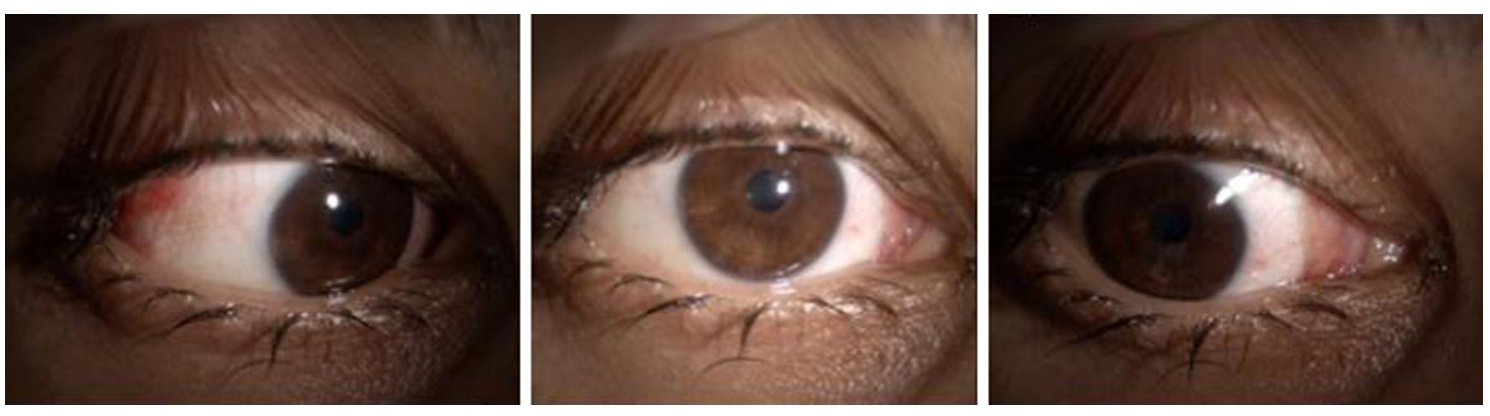

Fig. 1 First postoperative day after MISS with medial rectus plication and lateral rectus recession 

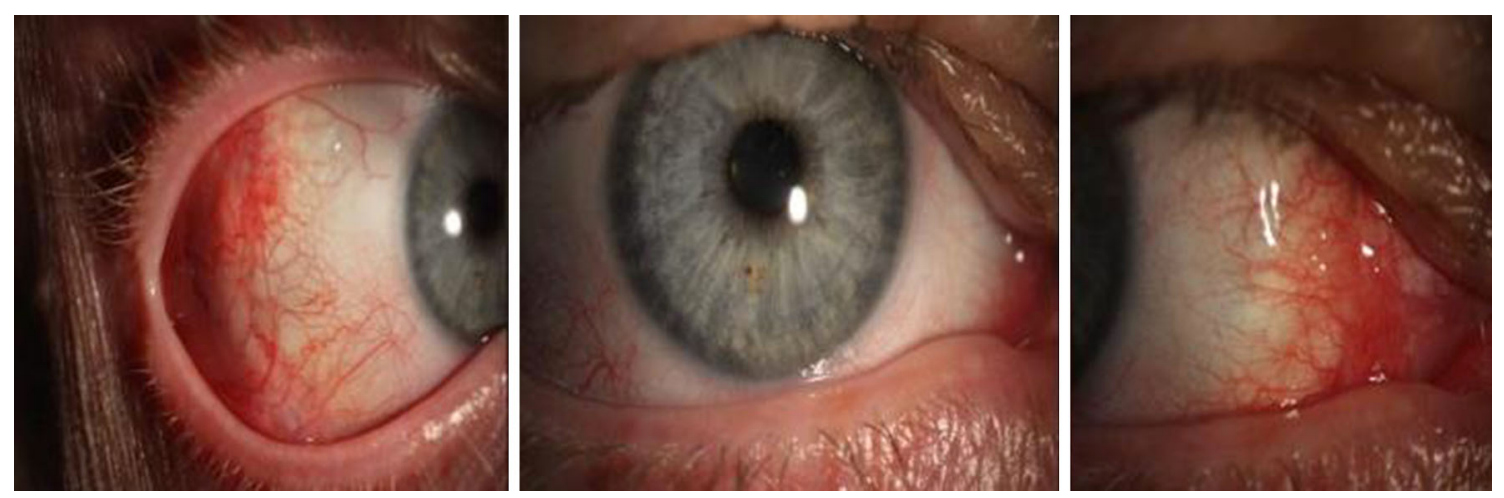

Fig. 2 Two weeks postoperatively after MISS with medial rectus recession and lateral rectus plication

the chances of scar formation and adhesions between the muscle and the overlying conjunctiva increase, thus hindering the dissection should repeat surgery become necessary. Muscle disinsertion should be done with careful attention to technique. If the cut is placed too close to the sclera, a permanently visible bluish line along the muscle insertion may ensue. If, on the other hand, the cut is placed too far from the sclera, the remaining tendon may form a visible elevation of the conjunctiva. In cases with hard to control bleeding this may necessitate keyhole enlargement that will allow adequate exposure for cauterization. Rinsing of blood should be done judiciously so that Tenon's swelling is minimized.

Occasionally, intraoperative conversion to a large conjunctival opening may become necessary. In a retrospective study with 982 rectus muscle procedures from 451 consecutive patients operated on by one surgeon over a time span of 5 years, Kaup and coworkers [14] evaluated the conversion rate of MISS to a conventional surgical limbal opening. The authors reported an overall conversion rate of 3.6\% (35/ 982) in their series. Twenty percent of these converted procedures $(7 / 35)$ were revisions, in the majority of which $(54 \%, 19 / 35)$ excessive intraoperative bleeding was recorded as the reason for conversion. Other reasons were insufficient visualization $(34 \%, 12 / 35)$ and significant scarring from previous operations $(11 \%, 4 / 35)$. It is worth noting, however, that the conversion rate decreased over time with increasing surgical experience from $8.4 \%$ in the first cases to $0.4 \%$ in the last cases. In addition, muscle resections were associated with a higher conversion rate $(P<0.001)$. Other factors like age, eye motility, primary versus revision surgery, and dose of correction did not significantly impact the likelihood of conversion [14].

According to Mojon [8], MISS is most suitable for patients between 14 and 40 years of age. In younger patients, excessive Tenon's capsule hinders the surgical approach to muscles, while in patients over 40 years of age poor conjunctival elasticity can lead to tears and the subsequent enlargement of microincisions. Overall, MISS can be employed in all types of strabismus surgeries, namely rectus muscle recessions, resections, plications, reoperations, retroequatorial myopexy, transpositions, oblique muscle recessions or plications, and adjustable sutures even in the presence of restricted motility [8, 15-21].

Future modifications of the originally described MISS approach may allow for even smaller incisions and further reduced tissue disruption. Indeed, Mojon [9] described a muscle reinsertion technique that employs transconjunctival suturing (TRASU) for horizontal rectus muscle recessions, plications, and advancements. Using the TRASU technique in 20 patients who underwent unilateral horizontal rectus surgery for primary displacements of at least $4.5 \mathrm{~mm}$, or repeat displacements of at least $3 \mathrm{~mm}$, this author observed that the technique was not associated with a higher rate of complications and the conjunctival incisions were eventually two-thirds the size of incisions used in standard MISS. Although the TRASU technique was originally described for 
horizontal rectus surgeries [9], it can be employed in all instances where muscle or sclera suturing is needed. The same author described a modified technique for rectus muscle plication in patients operated with MISS [17]. The technique is done without a spatula and thus, instead of total dissection (TODI), only marginal dissection (MADI) of the plicated portion of the rectus is performed. In a retrospective comparison of 40 patients operated on with the combined horizontal MISS rectus recession/MADI plication versus 49 matched patients who underwent MISS recession/TODI plication, the author found that the two techniques were equally effective and safe, but the group with MADI plication had significantly less swollen and erythematous conjunctivae postoperatively $(P<0.01)$.

\section{HOW TO PERFORM MISS IN STRABISMUS SURGERY}

\section{MISS for Primary Rectus Muscle Weakening and Strengthening $[8,17]$}

1. A limbal traction suture (e.g., 6-0 silk) is passed.

2. Two radial keyhole parainsertional cuts are made parallel to the upper and lower margin of the muscle. The length should be $1 \mathrm{~mm}$ shorter than the planned magnitude of the rectus muscle recession.

3. Small sub-Tenon tunnels joining the two incisions are made with Westcott scissors over the surface of the muscle, avoiding the muscular vessels.

4. The muscle is hooked and cauterization of the prominent blood vessels at the insertion underneath the conjunctiva is performed.

Continue for recession:

5. Vicryl sutures (6-0) are placed at the upper and lower poles of the muscle insertion, locked and secured. The sutures can then be preplaced at the desired recession site.

6. The muscle is cut at the insertion.

7. The muscle is carefully resutured at sclera at the planned position if not done so previously.

Continue for resection or plication:

8. Vicryl sutures (6-0) are passed at the upper and lower pole of the muscle at the planned position for resection or plication, locked and secured.

9. The sutures are placed through the muscle insertion ensuring an adequate anchoring scleral bite.

10. The muscle is divided in front of the suture level for resection or folded over for plication.

11. Tenon's capsule and conjunctiva are sutured with 8-0 vicryl sutures.

\section{MISS for Rectus Muscle Posterior Fixation} [20]

1. A corneal limbal traction suture is placed.

2. Two small L-shaped cuts are made, slightly anterior to the location where the scleromuscular sutures will be placed (4 $\mathrm{mm}$ long and $2 \mathrm{~mm}$ wide). If a concurrent recession or resection has been planned, then a 3-mm extension of the incision towards the limbus should be performed.

3. The episcleral tissue is carefully separated from the muscle sheath and the sclera using blunt scissors.

4. The location of the scleromuscular sutures is marked with a caliper.

5. On each muscle border a non-absorbable suture is passed through the sclera and one-third of the muscle, locked and secured.

6. Tenon's capsule and conjunctiva are sutured with 8-0 vicryl sutures.

\section{MISS for Oblique Muscle Recessions [19]}

1. A traction suture is placed.

2. A 4-mm incision is made over the muscle insertion (used for the disinsertion of the muscle).

3. A second incision is made over the planned point where the muscle is going to be anchored.

4. The episcleral tissue is separated from the muscle sheath and the sclera with blunt 
scissors. A sub-Tenon tunnel joining the two incisions is made.

5. A vicryl suture (e.g., 6-0) is passed at the insertion of the muscle, locked and secured.

6. The muscle is cut at its insertion.

7. A blunt sub-Tenon cannula $(20 \mathrm{G} \times 1)$ is passed through the tunnel.

8. The needle of the 7-0 vicryl suture is put into the cannula and the cannula is pulled back to take the needle out of the second incision.

9. The muscle is anchored on to the sclera at the planned point.

10. Tenon's capsule and conjunctiva are sutured with 8-0 vicryl sutures.

Undoubtedly MISS is currently the preferred approach of only a minority of surgeons worldwide and thus traditional techniques utilizing limbal or fornix conjunctival incisions remain most popular in many parts of the world [22]. It is worth noting, however, that the currently available published evidence has shown that strabismus surgery can indeed be performed safely and effectively through keyhole incisions with minimal collateral tissue damage [23]. Although the short-term advantage of faster rehabilitation and more satisfactory cosmesis can be important for some patients (Fig. 3), the real value of MISS lies in the long-term benefit of reduced fibrosis that will facilitate future reoperations should these be needed. The decreased likelihood of anterior chamber ischemia owing to the preservation of limbal blood vasculature is obviously an added
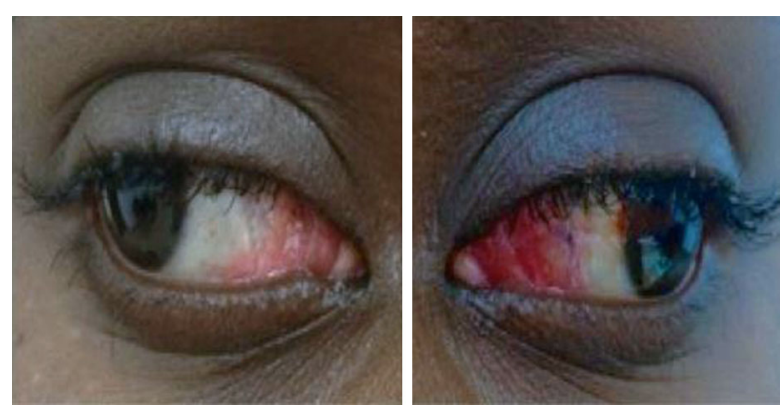

Fig. 3 One week postoperatively, bilateral medial rectus recession. The right eye was operated on with MISS, the left eye with a traditional fornix approach potential benefit of MISS. On the other hand, it is understandable that surgeons may be reluctant to adopt a novel, technically more challenging technique that has not yet proved superior to what is currently considered the gold standard. The existing literature comparing outcomes with MISS versus more traditional techniques is scarce. In a 6-month prospective study comparing patients operated on with MISS $(n=20)$ versus a matched, non-concurrent, retrospective comparison group $(n=20)$, Mojon [7] reported that no significant difference was detected for final ocular alignment, binocular single vision, visual acuity, refractive change, or complications. Sharma et al. [10] performed a parallel design randomized trial comparing MISS versus a paralimbal approach and examined conjunctival redness, congestion, chemosis, foreign body sensation, and topical medication intolerance. A total inflammatory score (TIS) derived from the sum of all previously mentioned scores was also calculated by the authors. Ocular alignment was not compared. The study included 28 eyes of 14 patients who were followed for up to 6 weeks. In each patient, one eye was randomized to MISS and the other to a standard paralimbal approach. After 6 weeks, eyes randomized to MISS had milder redness $(P=0.04)$ and better TIS $(P=0.05)$. Merino Sanz et al. [13] conducted a retrospective comparison of MISS versus a fornix approach in pediatric horizontal strabismus surgery and compared operating time, visual acuity, conjunctival hyperemia and swelling in the early postoperative period. Ocular alignment was not compared. The authors included 16 patients who were operated on with the MISS technique in one eye and the fornix approach in the fellow eye. The results indicated that the MISS technique was similar to the traditional fornix approach in the early postoperative period in terms of operating time, visual acuity, and conjunctival inflammation. Unfortunately, the critical outcome of ocular alignment using MISS has not been compared to that of more conventional techniques in randomized trials. Therefore, it remains to be proven that long-term outcomes using MISS are at least comparable to those using traditional techniques. It is clear then that more evidence 
from well-designed, controlled, long-term studies is needed in order to define the role, success rate, and limitations of MISS versus available options for strabismus surgery in different conditions and age groups.

\section{CONCLUSION}

Strabismus surgeons selecting MISS utilize keyhole conjunctival openings placed as far away from the limbus as possible in order to avoid damage to the perilimbal vessels and stem cells and reduce scarring around the muscle by avoiding excessive perimuscular dissection. The utilization of few keyhole incisions should lead to a more rapid recovery and reduction in postoperative complications such as conjunctival congestion and chemosis, corneal dellen, and Tenon's prolapse. The MISS technique optimizes the surgical management of strabismus and confers significant advantages as discussed above. However, it has the disadvantage of a long learning curve with increased surgical times initially. A higher incidence of scleral perforation with MISS has also been reported especially during the initial learning period. Thus, before starting to utilize MISS techniques a period of training next to an experienced MISS surgeon is highly recommended. In summary, MISS is a promising surgical approach that introduces the use of small incisions and reduced surgical manipulation in day-to-day strabismus surgery. It can improve outcomes and reduce complications by minimizing tissue disruption and preserving perilimbal vasculature. More controlled evidence, especially regarding alignment outcomes, is needed if it is to become the future gold standard in strabismus surgery.

\section{ACKNOWLEDGEMENTS}

No funding or sponsorship was received for the publication of this article.

All named authors meet the International Committee of Medical Journal Editors (ICMJE) criteria for authorship for this manuscript, take responsibility for the integrity of the work as a whole, and have given final approval for the version to be published.

Disclosures. Ioannis Asproudis, none; Nikolaos Kozeis, none; Andreas Katsanos has received honorariums from Santen and Allergan and had congress and travel expenses covered by Santen and Laboratoires Thea; Saurabh Jain, none; Paris G. Tranos, none; Anastasios G.P. Konstas has received honorariums from (and is a consultant for) Alcon, Allergan, Mundipharma, and Santen and had congress and travel expenses covered by MSD/Vianex.

Compliance with Ethics Guidelines. This article is based on previously conducted studies and does not involve any new studies of human or animal subjects performed by any of the authors.

Open Access. This article is distributed under the terms of the Creative Commons Attribution-NonCommercial 4.0 International License (http://creativecommons.org/licenses/ by-nc/4.0/), which permits any noncommercial use, distribution, and reproduction in any medium, provided you give appropriate credit to the original author(s) and the source, provide a link to the Creative Commons license, and indicate if changes were made.

\section{REFERENCES}

1. Swan KC, Talbott T. Recession under Tenon's capsule. Arch Ophthalmol. 1954;51:32-41.

2. Harms H. Uber Muskelvorlagerung. Klin Monatsbl Augenheilk. 1949;115:319-24.

3. Gobin $\mathrm{MH}$, Bierlaagh JJM. Chirurgie horizontale et cycloverticale simultanee du strabisme. Anvers: Centrum roor Strabologie; 1994.

4. Parks MM. Fornix incision for horizontal rectus muscle surgery. Am J Ophthalmol. 1968;65:907-15.

5. Velez G. Radial incision for surgery of horizontal rectus muscles. J Pediatr Ophthalmol Strabismus. 1980;17:106-7.

6. Gobin MH, Bierlaagh JJM. Chirurgie horizontale et cycloverticale simultanee du strabisme. Anvers: Centrum roor Strabologie; 1994. 
7. Mojon DS. Comparison of a new, minimally invasive strabismus surgery technique with the usual limbal approach for rectus muscle recession and plication. Br J Ophthalmol. 2007;91:76-82.

8. Mojon DS. Review: minimally invasive strabismus surgery. Eye (London). 2015;29:225-33.

9. Mojon DS. A new transconjunctival muscle reinsertion technique for minimally invasive strabismus surgery. J Pediatr Ophthalmol Strabismus. 2010;47:292-6.

10. Sharma R, Amitava AK, Bani SA. Minimally invasive strabismus surgery versus paralimbal approach: a randomized, parallel design study is minimally invasive strabismus surgery worth the effort? Indian J Ophthalmol. 2014;62:508-11.

11. Kushner BJ. Comparison of a new, minimally invasive strabismus surgery technique with the usual limbal approach for rectus muscle recession and plication. Br J Ophthalmol. 2007;91:5.

12. Merino P, Blanco Domínguez I, Gómez de Liaño P. Outcomes of minimally invasive strabismus surgery for horizontal deviation. Arch Soc Esp Oftalmol. 2016;91:69-73.

13. Merino Sanz P, de Liaño Gómez, Sánchez P, Domínguez IB. Minimally invasive strabismus surgery (MISS) compared with the fornix approach in pediatric horizontal strabismus surgery. Strabismus. 2015;23:159-63.

14. Kaup M, Mojon-Azzi SM, Kunz A, Mojon DS. Intraoperative conversion rate to a large, limbal opening in minimally invasive strabismus surgery (MISS). Graefes Arch Clin Exp Ophthalmol. 2011;249:1553-7.
15. Mojon DS. Minimally invasive strabismus surgery (MISS) for rectus muscle transpositions. Br J Ophthalmol. 2009;93:747-53.

16. Mojon DS. Minimally invasive strabismus surgery for horizontal rectus muscle reoperations. $\mathrm{Br} \mathrm{J}$ Ophthalmol. 2008;92:1648-52.

17. Mojon DS. A modified technique for rectus muscle plication in minimally invasive strabismus surgery. Ophthalmologica. 2010;224:236-42.

18. Mojon DS. Minimally invasive strabismus surgery for rectus muscle posterior fixation. Ophthalmologica. 2009;223:111-5.

19. Mojon DS. Minimally invasive strabismus surgery (MISS) for inferior obliquus recession. Graefes Arch Clin Exp Ophthalmol. 2009;247:261-5.

20. Pellanda N, Mojon DS. Combined horizontal rectus muscle minimally invasive strabismus surgery for exotropia. Can J Ophthalmol. 2010;45:363-7.

21. Pellanda N, Mojon DS. Minimally invasive strabismus surgery technique in horizontal rectus muscle surgery for esotropia. Ophthalmologica. 2010;224:67-71.

22. Mikhail M, Verran R, Farrokhyar F, Sabri K. Choice of conjunctival incisions for horizontal rectus muscle surgery-a survey of American Association for Pediatric Ophthalmology and Strabismus members. J AAPOS. 2013;17:184-7.

23. Granet DB, Wilson ME, Wagner RS. Minimally invasive strabismus surgery. J Pediatr Ophthalmol Strabismus. 2016;53:262-5. 